\title{
- Case report
}

\section{A report on a rare case of Kearns Sayre-like syndrome}

\author{
Gupta $\mathrm{Y}^{1}$, Gupta $\mathrm{M}^{2}$, Sambhav $\mathrm{K}^{3}$, Phougat $\mathrm{A}^{3}$,Varshney $\mathrm{A}^{3}$ \\ ${ }^{1}$ Professor, ${ }^{3}$ Resident, Institute of Ophthalmology \\ ${ }^{2}$ Reader, Department of physiology, JNMC \\ Aligarh Muslim University, Aligarh, India
}

\begin{abstract}
Purpose: To report the presentation of a rare case of Kearns-Sayre-like syndrome (KSS)

Case: A 14-year-old boy presented with progressive drooping of both upper eyelids for 10 years. Past history of right sided hemi-plegia and left facial nerve palsy was present. Examination (OU) showed total external ophthalmoplegia, ptosis and pigmentary retinopathy. The patient also had a short stature (height $121.9 \mathrm{~cm}$ ) for his age.
\end{abstract}

Keywords: Kearns Sayre syndrome, total external ophthalmoplegia, ptosis

\section{Introduction}

Kearns Sayre syndrome is the best known subtype of chronic progressive external ophthalmoplegia (CPEO) which is the most common mitochondrial disorder affecting muscles. The characteristic triad of this rare disease is total external ophthalmoplegia, pigmentry retinopathy and cardiac conduction defects during the first or second decade of life.

\section{Patient and methods}

The patient in our study reported to us in the routine OPD of J. N. M.C. Hospital, Aligarh, India. A 14year-old male presented to us with gradual drooping of both upper eyelids since 10 years. He had no history of difficulty in swallowing, muscular weakness, easy fatigability, sudden loss of consciousness, increase in drooping as the day progresses, diplopia, trauma or deafness. He had an episode of of right-sided hemiplegia and left facial nerve palsy at the age of 4 years which recovered fully without any specific treatment. He had no history of trauma, pain, redness, discharge or surgery to either eye. There was no history suggestive of any systemic disease or chronic illness in the past,

Received on: 03.01.2010 Accepted on: 16.04.2010

Correspondence and reprint request to:Prof Yogesh Gupata

B-6, Medical Colony, Aligarh UP 202002, India.

Tel: 0091-571-272 1202

Email: yogibear3190@hotmail.com similar complaints in the family or any history of consanguinity.

On examination, the patient had bilateral severe ptosis ( $5 \mathrm{~mm}, \mathrm{OU}$ ), poor LPS action of $4 \mathrm{~mm}$ (OU), forehead wrinkling, chin elevation, absent lid crease (Figure1) and absent bell's phenomenon. The corneal sensations were intact and ocular movements were absent in all directions of gaze (Figure 2). The pupils were of normal size and normally reactive to light and near reflex. Doll's eye movements were absent and orbicularis oculi weakness of both eyes was present. His best corrected visual acuity was $6 / 12$ with +0.50DS OU. Fundus examination (OU) showed pigmentary changes at the posterior pole.

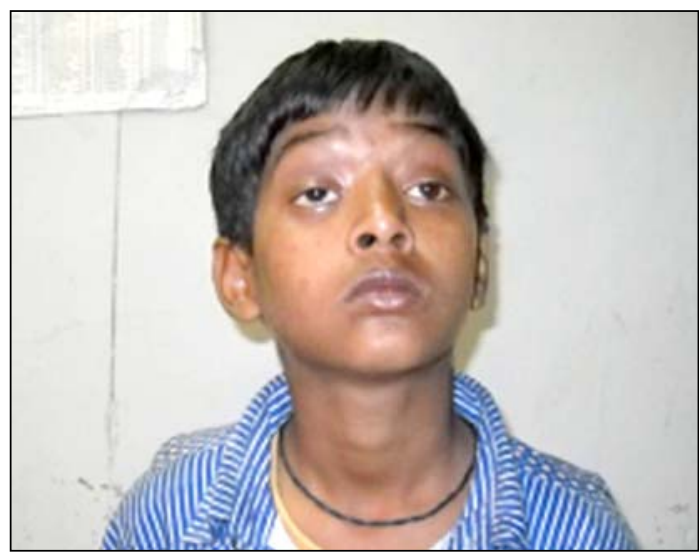

Fig 1: bilateral severe ptosis, forehead wrinkling, chin elevation and absent lid crease 


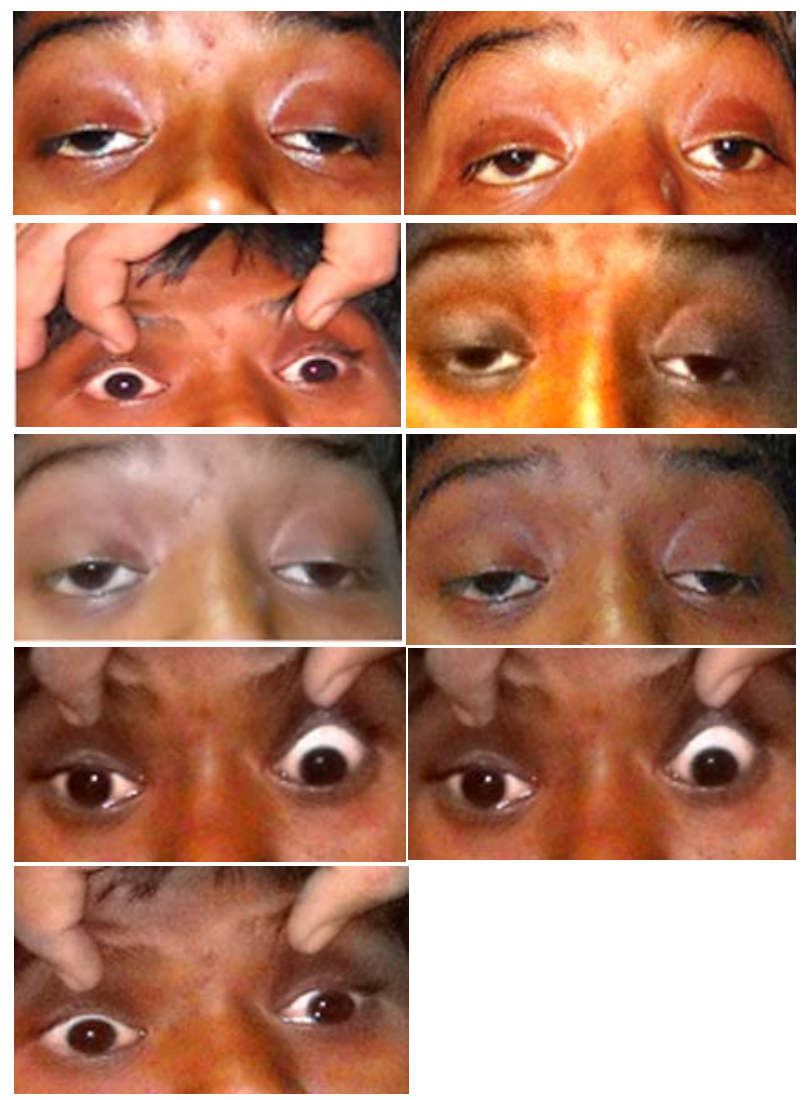

Fig 2: absence of ocular movements in all gazes.

The following investigations were done:

\section{TLC:}

Hemoglobin:

DLC:

ESR:

Blood Urea:

S. Creatinine:

Blood Sugar (F):

Blood Sugar (PP):

AEC:'

Urine $(\mathrm{R} \& \mathrm{M})$ :

Chest X- ray:

ECG:

MRI:



$12.0 \mathrm{~g} \mathrm{\%}$

8000 cells/cu.mm

N-58, L-32, B-0, M-0, E-10

$23 \mathrm{~mm}$ in I hour

$28 \mathrm{mg} \%$

$0.8 \mathrm{mg} \%$

$70 \mathrm{mg} \%$

$90 \mathrm{mg} \%$

$1000 / \mathrm{mm}^{3}$

WNL

WNL

WNL

Normal (Figure 3)

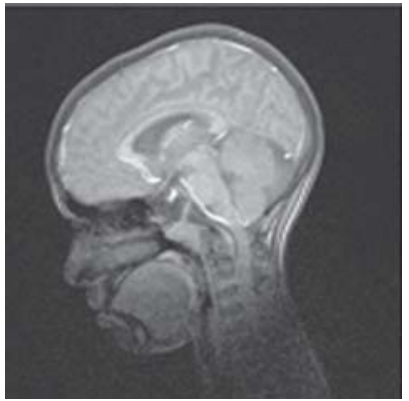

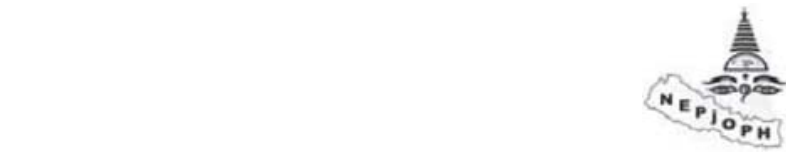
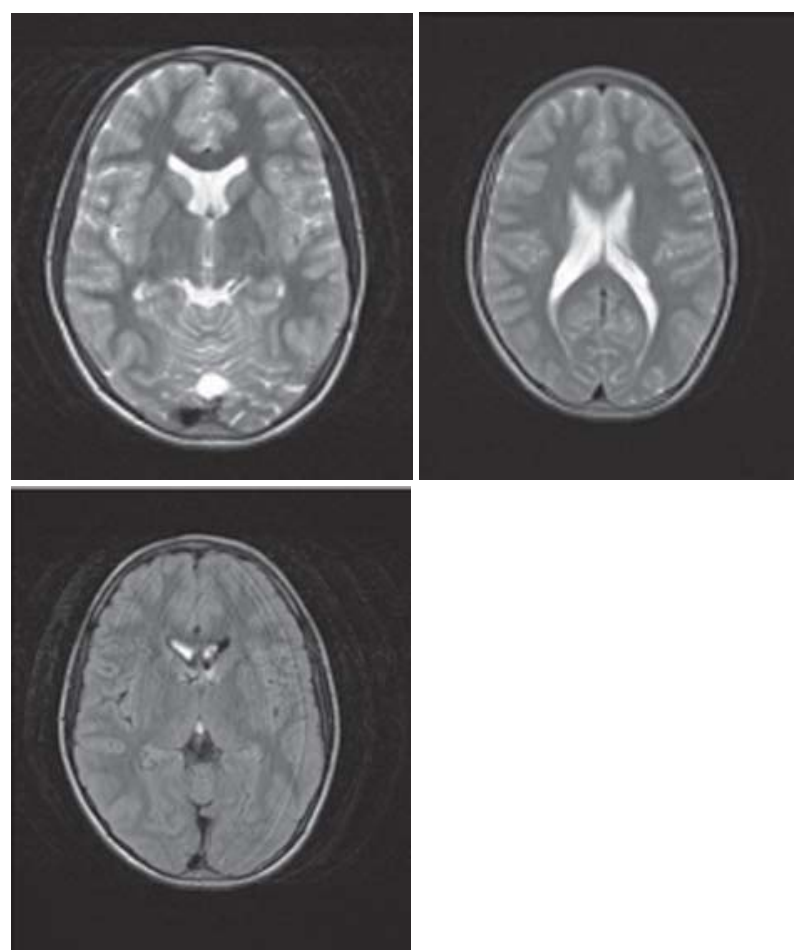

Fig 3

Further investigations that were recommended were: Serum lactate \& pyruvate CSF lactate \& protein

Muscle biopsy or blood leucocytes for histochemistry \& mitochondrial DNA analysis

Echocardiography

Electroretinography

Audiometry

Electromyography \& nerve conduction defects

Serum electrolytes, calcium, magnesium, cortisol \& thyroid function tests.

The patient left against medical advice and further evaluation could not be carried out.

\section{Discussion}

Mitochondria are cytoplasmic organelles that produce energy for cell functions, maintenance, repair, and growth through the enzymatic process of oxidative phosphorylation. A group of neurodegenerative and myopathic syndromes result from disorders of mitochondrial metabolism that cause defects in the energy cycle of susceptible tissues (Dimauro \& Moraes, 1993). Tissues most reliant on mitochondrial energy are CNS, heart, muscles, kidneys, and endocrine organs. Mitochondrial DNA is unique due 
to its cytoplasmic location, multiple copies in each cell, high mutation rate, and lacking the efficient repair mechanism present in nuclear DNA. Both wild type and mutant DNA are present in the same cell (heteroplasmy). Disease ensues when the proportion of wild/mutant DNA exceeds the tissue specific threshold. mtDNA is inherited almost exclusively through the maternal lineage, with only a single report of paternal inheritance (Schwartz \& Vissing, 2002).

Kearns Sayre Syndrome occurs as a result of largescale single deletions (or rearrangements) of mitochondrial DNA (mtDNA), which are usually not inherited but occur spontaneously, probably at the germ-cell level or very early in embryonic development(OMIM. Kearns-Sayre syndrome. Online Mendelian Inheritance in Man Web site). The risk of maternal transmission has been estimated to be approximately 1 in 24 (Chinnery PF et al 2004). Kearns-Sayre syndrome is a rare disorder. Marked heterogeneity and various types of inheritance have been observed. Remes et al (2005) estimated a prevalence of 1.6 cases per 100,000 population in a Finnish population ( 6 patients, only 3 of whom fulfilled the clinical criteria for Kearns-Sayre syndrome). Schaefer et al (2008) estimated a prevalence of 1.17 cases per 100,000 population of large-scale mitochondrial deletions in North East England; however, the proportion of patients with KearnsSayre syndrome is not stated (Schaefer et al 2008).

The most common mitochondrial disorder to affect muscles is chronic progressive external ophthalmoplegia (CPEO) and its best known subtype, Kearns Sayre syndrome. A few other known mitochondrial myopathies of ophthalmic importance are mitochondrial encephalopathy with lactic acidosis and stroke like syndrome (MELAS), myoclonic epilepsy with ragged red fibres (MERRE, fukuhara's syndrome), and mitochondrial neurogastrointestinal encephalopathy. Mitochondrial disorders that affect tissues other than muscle during early childhood include Alpers' disease, Menkes' disease, and Leigh's disease. Another variant, leber's hereditary optic neuropathy, presents later in life.

CPEO and KSS differ in the percentage and location of the mtDNA deletion (Lopez-Gallardo $\mathrm{E}$ et al 2009). CPEO is characterized by bilateral ptosis, external ophthalmoplegia and proximal myopathy. KSS is characterized by external ophthalmoplegia, pigmentary retinopathy and cardiac conduction block during the first or second decade of life (early onset CPEO) (Yanoff \& Duker, 2004). Affected children may have short stature, peripheral neuropathy, ataxia, spasticity, somatic muscle weakness, cognitive deficits (Bosbach S et al 2003) and multiple endocrinopathies like diabetes, hypoparathyroidism, growth hormone deficiency, hypogonadism and Addison disease. Bilateral sensorineural hearing loss is almost universal in those who survive into the fourth decade of life; this may not be fully corrected with hearing aids. Renal tubular acidosis (proximal or distal) has been described in numerous cases, with occasional progression to end-stage renal failure. Abnormalities of CNS include cerebellar dysfunction and elevated CSF protein exceeding $100 \mathrm{mg} / \mathrm{dl}$. Cardiac conduction defects have an onset typically 10 years after ptosis and may result in sudden death (Yanoff \& Duker, 2004). The brain may eventually undergo spongiform degeneration, with the clinical picture of dementia. Basal ganglia calcifications may occur. Biopsy of skeletal muscle reveals "ragged red fibres" that stain red or purple using a modified gomori trichrome stain.

Our case presented with an early onset CPEO (bilateral ptosis and total external ophthalmoplegia) and pigmentary retinopathy starting at 4 years of age with weakness of bilateral orbicularis oculi. CNS and endocrine involvement were characterized by an episode of hemiplegia with facial nerve palsy and short stature respectively. Cardiac conduction defects might occur at a later age as they typically occur 10 years after the onset of ptosis. Muscle biopsy could have diagnosed the case but the patient left against medical advice. This case represents a typical mitochondrial myopathic disorder most likely to be a rare Kearns-Sayre-like syndrome.

\section{References}

Bosbach S, Kornblum C, Schroder R, Wagner M (2003). Executive and visuospatial deficits in patients with chronic progressive external ophthalmoplegia and Kearns-Sayre syndrome. Brain.; 126(Pt 5):1231-40. 
DiMauro S, Moraes CT (1993). Mitochondrial encephalopathies. Arch Neurol.; 50:1197-207.

Lopez-Gallardo E, Lopez-Perez MJ, Montoya J, Ruiz-Pesini E (2009). CPEO and KSS differ in the percentage and location of the mtDNA deletion. Mitochondrion.; 9(5):314-7.

Moraes CT, DiMauro S, Zeviani M et al (1989). Mitochondrial DNA deletions in progressive external ophthalmoplegia and Kearns Sayre syndrome. N Engl J Med; 320:1293-9.

Remes AM, Majamaa-Voltti K, Karppa M et al (2005). Prevalence of large-scale mitochondrial
DNA deletions in an adult Finnish population. Neurology; 64(6):976-81.

Schaefer AM, McFarland R, Blakely EL et al (2008). Prevalence of mitochondrial DNA disease in adults. Ann Neurol.; 63(1):35-9.

Schwartz M, Vissing J (2002). Paternal inheritance of mitochondrial DNA. N Engl J Med; 347(8):576-80.

Yanoff M, Duker J S (2004). Ophthalmology, 2e, vol.2, 1342-3 